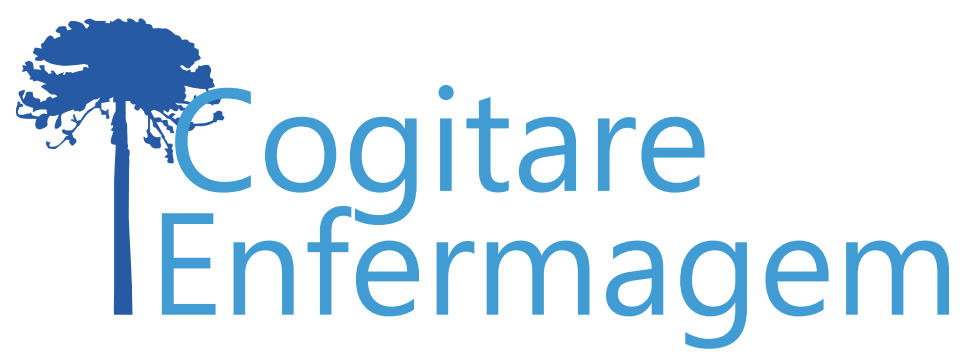

\title{
CRITÉRIOS DE ESCOLHA DE COBERTURAS PRIMÁRIAS NO TRATAMENTO DE LESÕES POR PRESSÃO EM PACIENTES HOSPITALIZADOS
}

\author{
Simone de Miranda Macêdo' ${ }^{1}$ \\ Letícia Lemos Ayres da Gama Bastos² \\ Rafaela Gomes Campello Oliveira ${ }^{3}$ (1) \\ Marize Conceição Ventin Lima² \\ Flávia Carolina Ferreira Gomes ${ }^{2}$ (1)
}

\begin{abstract}
RESUMO
Objetivo: analisar os critérios de escolha de coberturas primárias prescritas pelos enfermeiros para o tratamento de lesão por pressão em pacientes hospitalizados.

Método: estudo transversal e descritivo com abordagem quantitativa realizado em Hospital privado em Jaboatão dos Guararapes-Pernambuco, Brasil, entre fevereiro e abril de 2019. A amostra contou com 345 prontuários de pacientes com lesão por pressão, admitidos de janeiro a dezembro de 2018. Foi utilizado instrumento para coleta de dados sociodemográficos, realizadas análises descritivas e teste Qui-Quadrado com nível de confiança de 95\% $(p<0,05)$.

Resultados: houve relação significativa entre a escolha de coberturas e as características da lesão. Porém, 64 (18,5\%) pacientes da amostra continham registros adequados e 10 (3\%) apresentavam discordância com evidências científicas.

Conclusão: Esse estudo ressalta a importância da implantação de protocolos que ratifiquem os critérios de escolha das coberturas para o tratamento das lesões por pressão nos serviços hospitalares.
\end{abstract}

DESCRITORES: Ferimentos e Lesões; Lesão por Pressão; Pacientes Internados; Registros de Enfermagem; Qualidade da Assistência à Saúde.

\section{CRITERIOS DE SELECCIÓN DE APÓSITOS PRIMARIOS PARA EL TRATAMIENTO DE ÚLCERAS POR PRESIÓN EN PACIENTES HOSPITALIZADOS}

\section{RESUMEN:}

Objetivo: analizar los criterios de elección de las coberturas primarias prescritas por los enfermeros para el tratamiento de la lesión por presión en pacientes hospitalizados. Método: estudio transversal y descriptivo con enfoque cuantitativo realizado en un hospital privado de Jaboatão dos Guararapes-Pernambuco, Brasil, entre febrero y abril de 2019. La muestra incluyó 345 historias clínicas de pacientes con lesión por presión ingresados de enero a diciembre de 2018. Se utilizó un instrumento de recogida de datos sociodemográficos, análisis descriptivos y prueba de Chi-cuadrado con un nivel de confianza del 95\% ( $p<0,05)$. Resultados: hubo una relación significativa entre la elección de los apósitos y las características de la lesión. Sin embargo, 64 (18,5\%) pacientes de la muestra contenían registros adecuados y 10 (3\%) presentaban discordancia con la evidencia científica. Conclusión: Este estudio resalta la importancia de implantar protocolos que ratifiquen los criterios de elección de los apósitos para el tratamiento de las UP en los servicios hospitalarios.

DESCRIPTORES: Heridas y traumatismos; Úlcera por presión; Pacientes internos; Registros de enfermería; Calidad de la atención de salud. 
As lesões por pressão (LP) são caracterizadas por uma descontinuação da pele e tecidos moles subjacentes, denominadas de feridas, as quais podem ser comuns em proeminências ósseas ou provocadas por outros inúmeros fatores, podendo ser químicos, físicos ou biológicos ${ }^{(1)}$. O tecido tegumentar lesado inicia o processo de cicatrização de modo contínuo e complexo, o qual envolve fases sobrepostas para a recuperação da pele ${ }^{(2)}$.

As LP são classificadas por estágios, dependendo de fatores como profundidade da lesão, tempo de exposição e intensidade de pressão do tecido lesado, e sofrem forte influência de aspectos intrínsecos como hipertensão, diabetes mellitus, entre outros. Desta forma, as lesões vão desde o Estágio 1, no qual apresentam pele íntegra, mas com eritema não branqueável, até o Estágio 4, quando há perda total da espessura da pele e exposição ou palpação direta de tecidos como fáscia, músculo, tendão, ligamento, cartilagem ou osso". Existem ainda as LPP "Não Classificáveis", as quais possuem a perda tissular em sua espessura total, sendo difícil identificar a extensão da ferida, visto se encontrar encoberta pelo esfacelo ou escara ${ }^{(2)}$.

Atualmente o mercado oferece diversas marcas e tipos diferentes de cobertura que atuam em várias etapas do processo de cicatrização. Assim, o profissional, ao trabalhar com feridas, precisa reconhecer a lesão e saber indicar a melhor cobertura. A utilização de protocolos institucionais ajuda o profissional a ter segurança e garante a qualidade da assistência ${ }^{(3-4)}$.

Ademais, é essencial o registro das informações, no intuito de garantir a continuidade do cuidado e a avaliação dos resultados esperados. Tais informações devem ser sistemáticas e justificar a cobertura prescrita, além de conter as características da lesão, tipo de tecido, estágio de cicatrização(5). O enfermeiro é um profissional capacitado técnica e cientificamente, que possui uma atuação relevante no tratamento das lesões desde a avaliação, prescrição do tipo de cobertura até orientação de pacientes e familiares ${ }^{(6-7)}$.

Diante do importante papel desse profissional na manutenção e recuperação da integridade tissular, o presente estudo buscou analisar os critérios de escolha de coberturas primárias prescritas pelos enfermeiros para o tratamento de Lesão por Pressão em pacientes hospitalizados.

\section{MÉTODO}

Trata-se de um estudo transversal, descritivo, com abordagem quantitativa, que garante em uma ocasião, a partir de uma amostra adequada, examinar as distribuições de variáveis dentro dessa amostra, designando as variáveis preditoras e de desfecho com base na plausibilidade biológica e em informações de outras fontes ${ }^{(8)}$.

Para obtenção da amostra, utilizou-se o cálculo amostral para variáveis qualitativas em amostras finitas de forma aleatória simples, a qual foi constituída por 345 prontuários analisados no universo de 3.398 pacientes atendidos no ano de 2018. Para realização do cálculo do tamanho da amostra, utilizou-se a equação, para uma população finita: $\left.n=\left[z 2{ }^{*} p(1-p)\right] / e 2 / 1+\left[z{ }^{\star} p(1-p)\right] / e 2 * N\right]$. Na qual $n=$ tamanho da amostra; $N=$ tamanho da

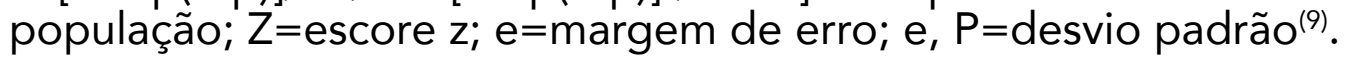

A coleta de dados foi realizada em um hospital, localizado em Jaboatão dos Guararapes-PE, entre os meses de fevereiro e abril de 2019. Para realização do estudo, considerou-se o seguinte critério de inclusão: prontuário eletrônico de pacientes admitidos 
entre janeiro e dezembro de 2018 e que apresentassem LP, admitidos nos setores de terapia intensiva, ortopedia, clínica médica ou unidade de cuidados prolongados.

O instrumento pré-estruturado elaborado pelos próprios pesquisadores buscou investigar as seguintes variáveis secundárias: dados sociodemográficos (sexo, faixa etária, estado civil, escolaridade), informações sobre histórico de doenças e as comorbidades associadas aos fatores de risco para cicatrização das LPP (hipertensão, diabetes, tabagismo, etilismo, acidente vascular encefálico, mobilidade prejudicada e desnutrição).

Quanto às características da lesão, foram identificados e registrados a partir do instrumento: tipo de ferimento (aguda ou crônica), agentes causadores, tempo de existência, tipo de tecido presente no leito do ferimento, localização, sinais de infecção, odor, quantidade de exsudato, aspecto da secreção e dor, e, por fim, a seleção do tipo de cobertura prescrita.

Para a tabulação dos dados, foi utilizado o Microsoft Excel 2013, e posteriormente, utilizou-se para a análise dos dados o software Statistical Package for the Social Sciences - SPSS v. 23, no qual foram realizadas as análises descritivas da população do estudo em valores relativos e absolutos, por meio de medidas de tendência central e distribuição de frequência. Além disso, foi realizado o teste Qui-Quadrado de Pearson para verificação da relação entre local e estágio da lesão, tipos de tecido presentes e as coberturas prescritas, considerando nível de confiança de $95 \%$ e valor de $\mathrm{p}<0,05$.

Os resultados foram apresentados por tabelas e discutidos a partir das evidências apresentadas pela diretriz do Guia de consulta rápida: prevenção e tratamento de úlceras por pressão(10).

O projeto foi submetido à Plataforma Brasil e os dados foram coletados após aprovação do comitê de ética da Faculdade Tiradentes de Jaboatão dos Prazeres, sob parecer n. 2.987.168.

\section{RESULTADOS}

No que se refere ao perfil sociodemográfico da amostra estudada ( $n=345)$, houve prevalência de indivíduos do sexo masculino $(n=173 ; 50 \%)$, que se enquadraram predominantemente na faixa etária de 61 a 80 anos $(n=181 ; 52 \%)$.

Em relação às comorbidades intrínsecas e às condições do paciente, de acordo com o diagnóstico médico e de enfermagem, como a causa/motivo/razão da hospitalização, destaca-se a Mobilidade Prejudicada (MP) com 161 (47\%), seguidos da Hipertensão Arterial Sistêmica (HAS) e do Diabetes Mellitus (DM) com 136 (40\%) e 114 (33\%), respectivamente. Foi identificado que essas comorbidades estavam mais presentes no sexo feminino, quando comparado ao sexo masculino, com 81 (51\%), 75 (55\%) e 66 (58\%), respectivamente, conforme demonstrado na Tabela 1.

Tabela 1 - Relação do Perfil sociodemográfico e comorbidade/condições dos pacientes em hospital público. Jaboatão dos Guararapes, PE, Brasil, 2018 (continua)

\begin{tabular}{ccccc} 
Variáveis & \multicolumn{2}{c}{ Feminino } & \multicolumn{2}{c}{ Masculino } \\
\cline { 2 - 5 } & $\mathrm{n}$ & $\%$ & $\mathrm{n}$ & $\%$ \\
\hline
\end{tabular}




\begin{tabular}{lcccc}
\hline 20 a 40 anos & 0 & 0 & 4 & 1 \\
\hline 41 a 60 anos & 9 & 2 & 16 & 5 \\
\hline 61 a 80 anos & 86 & 25 & 95 & 28 \\
\hline$>80$ anos & 77 & 22 & 58 & 17 \\
\hline Comorbidades/Condições & & & & \\
\hline Diagnóstico Médico & & & & \\
\hline Tabagismo & 1 & 11 & 8 & 89 \\
\hline Etilismo & 0 & 0 & 5 & 100 \\
\hline Hipertensão Arterial & 75 & 55 & 61 & 45 \\
\hline Diabetes Mellitus & 66 & 58 & 48 & 42 \\
\hline Acidente Vascular Encefálico & 37 & 45 & 46 & 55 \\
\hline Diagnóstico Enfermagem & & & & \\
\hline Mobilidade Prejudicada & 81 & 51 & 80 & 49 \\
\hline Desnutrição & 37 & 54 & 31 & 46
\end{tabular}

Fonte: Autores (2018)

Quanto à localização das LP, dos 345 prontuários analisados, apenas 317 apresentavam informações sobre a localização e estágio da lesão, dentre os quais foi possível perceber maior percentual na área sacrococcígea, principalmente no estágio II da lesão, o que correspondeu a 55 (22\%) das lesões totais, seguida da região do calcâneo em estágio III com quatro (14\%), conforme a Tabela 2.

Tabela 2 - Relação das variáveis "localização da lesão" X "estágio da lesão" em prontuários dos pacientes. Jaboatão dos Guararapes, PE, Brasil, 2018

\begin{tabular}{lcccccccccc} 
& \multicolumn{2}{c}{$\begin{array}{c}\text { Estágio } \\
\text { Iocal }\end{array}$} & \multicolumn{2}{c}{$\begin{array}{c}\text { Estágio } \\
\text { II }\end{array}$} & \multicolumn{2}{c}{$\begin{array}{c}\text { Estágio } \\
\text { III }\end{array}$} & \multicolumn{2}{c}{$\begin{array}{c}\text { Estágio } \\
\text { IV }\end{array}$} & \multicolumn{2}{c}{$\begin{array}{c}\text { Não } \\
\text { Classificável }\end{array}$} \\
\cline { 2 - 13 } & $\mathrm{n}$ & $\%$ & $\mathrm{n}$ & $\%$ & $\mathrm{n}$ & $\%$ & $\mathrm{n}$ & $\%$ & $\mathrm{n}$ & $\%$ \\
\hline Sacrococcígea & 12 & 5 & 55 & 22 & 33 & 13 & 6 & 1 & 5 & 2 \\
\hline Calcâneo & 0 & 0 & 2 & 7 & 4 & 14 & 1 & 3 & 1 & 3 \\
\hline Trocânter & 2 & 7 & 2 & 7 & 1 & 4 & 1 & 4 & 0 & 0 \\
\hline Membro inferior direito & 0 & 0 & 0 & 0 & 0 & 0 & 0 & 0 & 0 & 0 \\
\hline Membro inferior esquerdo & 0 & 0 & 1 & 20 & 0 & 0 & 0 & 0 & 0 & 0 \\
\hline Maléolo & 0 & 0 & 2 & 28 & 0 & 0 & 0 & 0 & 0 & 0 \\
\hline Outras Regiões & 1 & 2 & 11 & 25 & 3 & 7 & 0 & 0 & 0 & 0
\end{tabular}

Fonte: Autores (2018) 
Em relação às variáveis "tipo de tecido" e "coberturas utilizadas", dos 345 prontuários, foi verificado que apenas 79 (22,89\%) continham algum tipo de informação referente às características da lesão e apenas $64(18,5 \%)$ justificavam a partir dos registros os critérios de escolha da cobertura. Desses 64 prontuários com as informações registradas, foi possível identificar a escolha das coberturas prescritas para cada tipo de tecido, conforme apresentado na Tabela 3. É importante ressaltar que a mesma lesão pode apresentar mais de um tecido em sua extensão, o que justifica a necessidade da prescrição de mais de uma cobertura por lesão encontrada.

Tabela 3 - Relação das variáveis "tipo de tecido" e "coberturas utilizadas" nas lesões nos prontuários dos pacientes. Jaboatão dos Guararapes, PE, Brasil, 2018

\begin{tabular}{|c|c|c|c|c|c|c|c|c|c|c|}
\hline & \multicolumn{2}{|c|}{ AGE* } & \multicolumn{2}{|c|}{ Colagenase } & \multicolumn{2}{|c|}{ Hidrogel } & \multicolumn{2}{|c|}{$\begin{array}{l}\text { Carvão } \\
\text { ativado }\end{array}$} & \multicolumn{2}{|c|}{$\begin{array}{l}\text { Alginato de } \\
\text { cálcio }\end{array}$} \\
\hline & $\mathrm{n}$ & $\%$ & $\mathrm{n}$ & $\%$ & $\mathrm{n}$ & $\%$ & $\mathrm{n}$ & $\%$ & $\mathrm{n}$ & $\%$ \\
\hline \multicolumn{11}{|l|}{ Tipo Tecido } \\
\hline Hiperemia & 1 & 14 & 2 & 20 & 1 & 5 & 0 & 0 & 0 & 0 \\
\hline Tecido & 4 & 57 & 4 & 40 & 4 & 19 & 0 & 0 & 2 & 17 \\
\hline \multicolumn{11}{|l|}{ Granulação } \\
\hline Tecido Necrótico & 4 & 57 & 7 & 70 & 13 & 62 & 7 & 10 & 4 & 33 \\
\hline Tecido Esfacelo & 3 & 43 & 1 & 10 & 8 & 38 & 3 & 9 & 4 & 33 \\
\hline
\end{tabular}

${ }^{*}$ AGE $=$ Ácido Graxos Essenciais

Fonte: Autores (2018)

O Ácidos Graxos Essenciais (AGE) foi prescrito para todos os tipos de tecido, no qual se constatou o seguinte percentual: tecido hiperemiado um (14\%), tecido de granulação quatro (57\%), tecido necrótico quatro (57\%) e tecido de esfacelo três (43\%).

A prescrição da colagenase foi registrada principalmente na presença de tecido necrótico em sete (70\%), em lesões com esfacelo um (10\%), em tecido de granulação quatro (40\%) e em lesões caracterizadas por hiperemia dois (20\%).

Já o uso do hidrogel esteve presente em todos os tipos de tecidos, sendo prescrito uma vez $(5 \%)$ para tecido com hiperemia, quatro $(19 \%)$ em tecido de granulação e, principalmente para os tecidos desvitalizados como o tecido necrótico, em que foi utilizado 13 vezes (62\%), e oito (38\%) em tecido de esfacelo.

O carvão ativado foi escolhido sete vezes (10\%) como cobertura em tecido necrótico, três vezes (9\%) no tecido de esfacelo e 10 vezes (42\%) nas lesões com odor fétido. O alginato de cálcio foi utilizado duas vezes em lesões com tecido de granulação (17\%), quatro vezes (33\%) em tecido necrótico e quatro vezes (33\%) em tecido de esfacelo. Foi possível perceber que 15 (3\%) prontuários estavam em discrepância com a literatura no que se refere às características da lesão e escolha da cobertura.

O presente estudo verificou a associação entre as variáveis "tipo de tecido" e "coberturas utilizadas", como pode ser visto na Tabela 4, por meio do teste de quiquadrado. Observou-se associação significativa para as variáveis: AGE em tecido de granulação $(p=0,000)$, bem como em tecido necrótico $(p=0,011)$; colagenase em tecido 
necrótico $(p=0,000)$ e em tecido de granulação $(p=0,000)$; hidrogel em tecido de granulação $(p=0,019)$, em tecido necrótico $(p=0,000)$ e em tecido de esfacelo $(p=0,000)$; e, por fim, carvão ativado em tecido necrótico $(p=0,032)$.

Tabela 4 - Descrição do teste qui-quadrado para as coberturas relacionadas ao tipo de tecido utilizado nas lesões nos prontuários de pacientes. Jaboatão dos Guararapes, PE, Brasil, 2018

\begin{tabular}{lccccc} 
& AGE & Colagenase & Hidrogel & $\begin{array}{c}\text { Carvão } \\
\text { ativado }\end{array}$ & $\begin{array}{c}\text { Alginato de } \\
\text { cálcio }\end{array}$ \\
\hline Hiperemia & 0,829 & 0,198 & 0,509 & 0,179 & 0,277 \\
\hline Tecido Granulação & $0,000^{*}$ & $0,000^{*}$ & $0,019^{*}$ & 0,244 & 0,157 \\
\hline Tecido Necrótico & $0,035^{\star}$ & $0,000^{*}$ & $0,000^{*}$ & $0,032^{\star}$ & 0,215 \\
\hline Tecido Esfacelo & 0,114 & 0,795 & $0,000^{*}$ & 0,713 & 0,246
\end{tabular}

*Dados estatisticamente significativos, com valores $\mathrm{p}<0,05$.

Fonte: Autores (2018)

\section{DISCUSSÃO}

Dentre as comorbidades e fatores de riscos associados à LP, a mobilidade prejudicada se apresentou em maior percentual na amostra estudada. $O$ grau de limitação física interfere de forma direta na capacidade do indivíduo executar atividades diárias, podendo causar predisposição para o desenvolvimento das LP(11). Nestas situações em que há limitação da deambulação, a região sacra é o local mais acometido e sua incidência pode variar de $29,51 \%$ a $35,84 \%$, seguido das regiões do calcâneo e trocantérica, devido à permanência do paciente em decúbito dorsal e à compressão do tecido em proeminência óssea, consequentemente, diminuindo a capacidade do fluxo sanguíneo e favorecendo hipóxia dérmica ${ }^{(12)}$.

A presença de comorbidades e idade avançada da amostra analisada neste estudo pode corroborar com a literatura no que se refere à presença dos fatores de risco. Foram identificados, além da mobilidade prejudicada, principalmente a diabetes mellitus e a hipertensão como causadores do retardamento ou impedimento do avanço do processo de cicatrização das LP nos pacientes devido à alteração na perfusão tecidual e consequente possibilidade de isquemia(13).

Aseleção da cobertura deve ser baseada nos seguintes critérios de escolha: capacidade de manter o leito da lesão úmido, abordagem bacteriana, natureza e volume do exsudato da lesão, condição do tecido no leito da lesão, tamanho, profundidade e localização, além da presença de tunelizações e/ou cavitações ${ }^{(10)}$. A falta de registros adequados encontrada nos dados analisados pode ser indicativo de que esses critérios não estão sendo levados em consideração na hora da escolha da cobertura, além de dificultar o acompanhamento da evolução da lesão.

Em relação à utilização das coberturas primárias, o AGE apresenta uma grande efetividade nas lesões relacionadas à pele, infectada ou não, promovendo a angiogênese a epitelização, a fim de manter o meio úmido e favorecer o processo de granulação tecidual. Sua aplicação na pele íntegra tem grande absorção, pois forma uma película protetora, 
apresentando alta capacidade de hidratação e proporcionando uma nutrição celular local(1). A ampla possibilidade de utilização do AĢE explica a prescrição em vários tipos de tecidos encontrados nos prontuários analisados.

Porém, apesar de não haver contraindicações, não há evidências de resultados na literatura quanto à eficácia da cobertura $A G E$ no que se refere à angiogênese em tecidos desvitalizados ${ }^{(3)}$, como encontrado neste estudo, o que pode retardar o tempo de cicatrização e prolongar o de internação, com aumento significativo dos custos relacionados ao tratamento(14). É necessário ressaltar que neste estudo havia a possibilidade de uso de coberturas com eficácia científica comprovada para remoção de tecido desvitalizado, como colagenase e hidrogel.

A colagenase apresenta um grande potencial enzimático que favorece a remoção do tecido desvitalizado, independentemente de sua origem e localização, sendo potencialmente eficaz no desbridamento das células de colágeno e devendo permanecer por até 24 horas. Apesar de a eficácia da colagenase em tecidos de granulação e epitelial ainda gerar dúvidas, uma vez que o aumento das proteínas acaba ocasionando a degradação da membrana celular ${ }^{(3)}$, a prescrição dessa esteve presente em tecido com hiperemia e de granulação nos prontuários analisados, o que pode acarretar em prejuízo no tempo de cicatrização da lesão e ser indicativo da necessidade de investimento na educação permanente dos profissionais, para garantir a prática baseada em evidências e a qualidade da assistência prestada.

Tanto para o desbridamento autolítico como para a cicatrização do tecido de granulação, houve a disponibilização da cobertura hidrogel nos prontuários analisados. Essa cobertura é um gel transparente incolor composto por água, carboximetil celulose, que facilita a reidratação celular e o seu desbridamento, e propilenoglicol, que tem a capacidade de absorver água e hidratar a pele, promovendo a liberação de exsudato e a hidratação no tecido, além de ação bacteriostática ${ }^{(15)}$. Ela deverá permanecer na lesão por até 72 horas fazendo o desbridamento através da promoção do meio úmido, estimulando a migração dos leucócitos e ação de enzimas ${ }^{(16)}$.

Nos casos de lesões neoplásicas ou infeccionadas, o carvão ativado deverá ser utilizado a fim de absorver o exsudato e filtrar os odores desagradáveis, exercendo uma função bactericida por conta da ação da prata e consequentemente controlando a infecção(1). Deve ser trocado a cada 24 horas a depender da capacidade de adsorção, após a melhora da lesão, devendo ser trocado no máximo em 48 ou 72 hora $^{\text {s(17) }}$. Essa indicação explica a presença dessa cobertura, ainda que em pequeno percentual, em lesões com tecido necrótico e esfacelo nos resultados encontrados.

O uso do alginato de cálcio é sugerido pela literatura, tanto na presença de tecido de granulação quanto em tecido necrótico, visto que apresenta propriedades hemostáticas, além de facilitar o desbridamento autolítico, promovendo a estimulação do tecido de granulação ${ }^{(7)}$. Dessa forma, o alginato de cálcio, prescrito em diferentes tipos de lesões neste trabalho, está indicado nos ferimentos abertos, altamente absorvente e biodegradável derivado de material absorvente não tecido, com ou sem o aparecimento de infecções e facilitando o estímulo rápido de tecido de granulação.

A troca do alginato deve ser realizada a cada 24 horas nos ferimentos e lesões considerados infectados e a cada 72 horas nos necrosados ${ }^{(16)}$. O tratamento por alginato de cálcio como cobertura primária tem por objetivo o desbridamento, apresentando-se eficaz em relação às LP não classificáveis e granuladoras em estágio III e IV ${ }^{(17)}$. Assim, realizar o registro sistemático da avaliação e do tipo de cobertura utilizado no tratamento das lesões constitui-se o elemento fundamental para garantir uma assistência de enfermagem qualificada.

Observou-se neste estudo que os enfermeiros não descrevem as características clínicas das lesões, ou seja, não especificam o seu tamanho, característica da pele, quantidade de exsudato, odor, tipo de tecido presente na lesão e dor. Tais dados são de extrema 
importância, visto que interferem na escolha específica do tipo de cobertura, bem como na cicatrização das lesões ${ }^{(12)}$. A falta de registro das características da lesão nos prontuários ou registros incompletos e genéricos gera parâmetros insuficientes para o acompanhamento dos resultados e pode comprometer a assistência prestada ao paciente ${ }^{(18-19)}$.

Os profissionais de enfermagem não estão conscientizados sobre a relevância da escrita, como o registro do cuidado realizado de forma metódica; em consequência, observase utilização de frases e termos tanto repetitivos como vagos, os quais não refletem a totalidade da assistência prestada ${ }^{(20)}$. O registro da avaliação, evolução e curativo utilizados na realização da troca do tipo de cobertura, possibilitam a continuidade do cuidado e a realização de estatísticas dos diversos atendimentos, servindo também como fonte de consulta e documentos legais, que favorecem a melhoria da qualidade da assistência ${ }^{(7)}$.

Essa ausência nas anotações de enfermagem foi a maior limitação neste estudo, pois dificultou a análise mais detalhada das potencialidades e fragilidade do serviço no tratamento das lesões e, consequentemente, na eficiência das coberturas utilizadas.

\section{CONCLUSÃO}

Evidenciou-se a ausência de registro das evoluções de enfermagem nos prontuários dos pacientes; mesmo havendo instrumento específico para acompanhamento das LP no hospital, esse não é utilizado por todos os profissionais, o que dificulta o planejamento e a avaliação da qualidade da assistência.

Foi possível identificar prescrições que vão de encontro às evidências científicas e com critérios inconsistentes. Esses achados ressaltam a importância da implantação de um protocolo claro que ratifique os critérios de escolha das coberturas primárias pelos enfermeiros.

\section{REFERÊNCIAS}

1. Associação Brasileira de Estomaterapia, Seção São Paulo. Classificação das lesões por pressão consenso NPUAP 2016. São Paulo: SOBEST (SP); 2016 [acesso em 30 jul 2020]. Disponível em: http:// www.sobest.org.br/textod/35.

2. Silva AC de O, Rodrigues Filho E de S, Sousa GR da S, Silva JF dos S e, Silva AL da, Araújo CM dos $S$. As principais coberturas utilizadas pelo enfermeiro. Revista UNINGÁ [Internet]. 2017 [acesso em 16 jun 2019]; 53(2). Disponível em: http://revista.uninga.br/index.php/uninga/article/view/1426/1041.

3. Silva MJS. Protocolo de cuidados e tratamento para lesão por pressão [monografia]. Rio Grande do Norte (RN): Faculdade de Ciências da Saúde do Trair; 2018.

4. Abreu MA, Rolim VE, Dantas RC de O. Ações de enfermagem para prevenção de úlceras por pressão em clientes em unidade de terapia intensiva. Rev. Pesq. Int. [Internet]. 2017 [acesso em 04 jun 2020]; 2(suppl). Disponível em: http://revistas.ufcg.edu.br/cfp/index.php/pesquisainterdisciplinar/article/ view/400/pdf.

5. Prado AR de A, Barreto VPM, Tonini T, Silva AS da, Machado WCA. O Saber do enfermeiro na indicação de coberturas no cuidado ao cliente com feridas. ESTIMA [Internet]. 2016 [acesso em 16 jun 2019]; 14(4). Disponível em: http://dx.doi.org/10.5327/Z1806-3144201600040004.

6. Conselho Federal de Enfermagem. Lei n. 5.905, de 12 julho 1973. Dispõe sobre as normas técnicas 
que regulamenta a atuação da equipe de enfermagem no cuidado aos pacientes com feridas. Brasília: COFEN; 2018.

7. Freitas, TS. A importância do enfermeiro na avaliação e tratamento de feridas [monografia]. São Paulo (SP): Faculdade de Pindamonhangaba; 2017.

8. Hulley SB, Cummings SR, Browner WS, Grady DG, Newman TB. Delineando a pesquisa clínica. Porto Alegre: Artmed; 2015.

9. Gil, AC. Métodos e técnicas de pesquisa social. São Paulo: Atlas; 2019.

10. National Pressure Ulcer Advisory Panel, European Pressure Ulcer Advisory Panel, Pan Pacific Pressure Injury Alliance. Prevention and Treatment of Pressure Ulcers: Quick Reference Guide. [Internet]. Osborne Park: Cambridge Media; 2014 [acesso em 30 jul 2020]. Disponível em: https://www.epuap.org/wpcontent/uploads/2010/10/Quick-Reference-Guide-DIGITAL-NPUAP-EPUAP-PPPIA-16Oct2014.pdf.

11. Cândido KP, Souza JC de, Oliveira FM de. Perfil das pessoas com lesão por pressão na reabilitação: relação entre braden e dependência funcional. Rev. Enferm. Atual [Internet]. 2019 [acesso em 17 jun 2019]; 87(25). Disponível em: https://doi.org/10.31011/reaid-2019-v.87-n.especial-art.164.

12. Santos, RM. A importância da aplicação da escala de braden na prevenção das lesões por pressão em pacientes da unidade de terapia intensiva [monografia]. São Paulo (SP): Faculdade Anhanguera de Santa Bárbara d'Oeste; 2018.

13. Oliveira VC, Constante SAR. Lesão por pressão: uma revisão de literatura. Revista Psicologia e Saúde em Debate [Internet]. 2018 [acesso em 17 jun 2019]; 4(2). Disponível em: https://doi.org/10.22289/2446922X.V4N2A6.

14. Donoso MTV, Barbosa SAS, Simino GPR, Couto BRGM, Ercole FF, Barbosa JAG. Análise de custos do tratamento de lesão por pressão em pacientes internados. Rev. enferm. Cent.-Oeste. [Internet]. 2019 [acesso em 04 jun 2020]; 9:e3446. Disponível em: http://doi.org/10.19175/recom.v9i0.3446.

15. Geovanini T, Oliveira Junior AG de. Manual de curativos. São Paulo: Corpus; 2015.

16. Fontes $F L$ de $L$, Oliveira $A C$. Competências do enfermeiro frente à avaliação e ao tratamento de feridas oncológicas. Revista UNINGÁ [Internet]. 2019 [acesso em 18 jun 2019]; 56(2). Disponível em: http://revista.uninga.br/index.php/uninga/article/view/2158.

17. Ramos AF, Pinto MCPF. Dermatite associada à incontinência em pacientes portadores de doenças crônicas. Revista UNINGÁ [Internet]. 2016 [acesso em 17 jun 2019]; 47(2), Disponível em: http://revista. uninga.br/index.php/uninga/article/view/1265/887.

18. Gardona RGB, Ferracioli MM, Salomé GM, Pereira MT de J. Avaliação da qualidade dos registros dos curativos em prontuários realizados pela enfermagem. Rev Bras Cir Plást [Internet]. 2013 [acesso em 16 jun 2019]; 28(4). Disponível em: http://www.rbcp.org.br/details/1460/avaliacao-da-qualidade-dosregistros-dos-curativos-em-prontuarios-realizados-pela-enfermagem.

19. Galdino Júnior H, Tipple AFV, Lima BR de, Bachion MM. Processo de enfermagem na assistência a pacientes com feridas em cicatrização por segunda intenção. Cogitare enferm. [Internet]. 2018 [acesso em 04 jun 2020]; 23(4). Disponível em: http://dx.doi.org/10.5380/ce.v23i4.56022.

20. Aquino M de JN de, Cavalcante T de MC, Abreu RNDC de, Scopacasa LF, Negreiros FD da S. Anotações de enfermagem: avaliação da qualidade em unidade de terapia intensiva. Enferm. Foco. [Internet]. 2018 [acesso em 04 jun 2020]; 9(1). Disponível em: http://revista.cofen.gov.br/index.php/ enfermagem/article/view/1314/419. 
COMO REFERENCIAR ESTE ARTIGO:

Macêdo S de M, Bastos LLA da G, Oliveira RGC, Lima MCV, Gomes FCF. Critérios de escolha de coberturas primárias no tratamento de lesões por pressão em pacientes hospitalizados. Cogitare enferm. [Internet]. 2021 [acesso em "colocar data de acesso, dia, mês abreviado e ano"]; 26. Disponível em: http://dx.doi.org/10.5380/ ce.v26i0.74400.

Recebido em: 08/06/2020

Aprovado em: 11/12/2020

Editora associada: Luciana Alcântara Nogueira

Autor Correspondente:

Flávia Carolina Ferreira Gomes

Faculdade de Comunicação e Turismo de Olinda - Olinda, PE, Brasil

E-mail: fcferreiragomes@gmail.com

Contribuição dos autores:

Contribuições substanciais para a concepção ou desenho do estudo; ou a aquisição, análise ou interpretação de dados do estudo - SMM, LLAGB, FCFG

Elaboração e revisão crítica do conteúdo intelectual do estudo - SMM, LLAGB, RGCO, MCVL, FCFG

Aprovação da versão final do estudo a ser publicado - SMM, LLAGB, RGCO, MCVL, FCFG

Responsável por todos os aspectos do estudo, assegurando as questões de precisão ou integridade de qualquer parte do estudo - SMM, FCFG

Copyright $\odot 2021$ Este é um artigo em acesso aberto distribuído nos termos da Licença Creative Commons Atribuição, que permite o uso irrestrito, a distribuição e reprodução em qualquer meio desde que o artigo original seja devidamente citado. 